\title{
Extractivismo agroindustrial en zonas de colonización cocalera, análisis en el municipio de Mapiripan (Meta, Colombia)
}

\section{Agro-industrial extractivism in areas of cocalera colonization, analysis in the municipality of Mapiripan (Meta, Colombia)}

\author{
CASTRO-GARZÓN, Hernando ${ }^{1}$ \\ LOPEZ, Alvaro A. ${ }^{2}$ \\ RODRIGUEZ, Juan P. ${ }^{3}$
}

\begin{abstract}
Resumen
Se realiza un análisis de la influencia del extractivismo agroindustrial en el municipio de Mapiripan, Meta, Colombia, posterior a la bonanza de cultivos de uso ilicito finalizada en 2009. Las zonas de colonización cocalera del sur del Meta se caracterizan por el aislamiento social y geográfico, al establecerse la industria crea un nuevo modelo económico de extracción con impactos diversos. Se uso un método cualitativo explicativo, definiendo historicamente las intervenciones de los dos modelos ejercidos en la zona.

Palabras clave: sostenibilidad, desarrollo local, extractivismo
\end{abstract}

\begin{abstract}
An analysis of the influence of the agroindustrial extractivism in the municipality of Mapiripan, Meta, Colombia, was carried out following the bonanza of crops of ilicite use completed in 2009. The coca-bearing colonization zones of the south of Meta are characterized by social and geographical isolation, when the industry is established it creates a new economic model of extraction with diverse impacts. An explanatory qualitative method is used, defining historically the interventions of two models exercised in the area.

key words: sustainability, local development, extractivism
\end{abstract}

\section{Introducción}

Los programas de desarrollo local en las zonas de colonización cocalera se han enfocado principalmente en la sustitución de cultivos de uso ilícito por proyectos de economía campesina. Asi mismo, se ha asignado un rol central a los organismos gubernamentales en la provisión de la infraestructura económica y social. En el municipio de Mapiripán a esta realidad se traslapa la incursión de grandes capitales privados que por medio de un modelo extractivista ${ }^{4}$ han impactado notoriamente en la gestión del desarrollo local, y se han constituido en

\footnotetext{
${ }^{1}$ Profesor. Facultad de Ciencias Ecónomicas. Universidad de los Llanos, hcastro@unillanos.edu.co

2 Maestrante. Estudios de Desarrollo Local. Universidad de los Llanos, alvaromichin@gmail.com

${ }^{3}$ Profesor Titular. Facultad de medio Ambiente y Recursos Naturales. Universidad Distrital Francisco Jóse de Caldas, jprodriguezm@udistrital.edu.co

${ }^{4}$ Es necesario distinguir dos tipos de «extractivismo». El primero se origina en los sectores minero y energético, y comprende la explotación de metales preciosos, petróleo, gas y minerales, que se destinan principalmente a la exportación. El segundo se genera en el sector agrícola, y comprende extensas
} 
una opción para la superación de los cultivos de uso ilícito. En esta coyuntura, es menester reconocer las dificultades históricas para lograr el desarrollo local en las zonas de colonización cocalera, debido a su prolongado aislamiento geográfico, social y económico. Para comprender los elementos causales y sus efectos se aborda la relación entre desarrollo local y extractivismo desde diferentes perspectivas.

Por un lado, la tradición critica latinoamericana en contra de los grandes proyectos extractivistas, especialmente del modelo de enclave exportador agro-minero, encuentra referencias intelectuales en las obra de (Cardoso \& Faletto, 1996; Frank, Pacios \& Izaguirre, 1970; Furtado, 1971) quienes, en pocas palabras, sostienen que los efectos positivos del extractivismo sobre las sociedades locales, los mercados de trabajo y la naturaleza son nulos. (Alimonda, 2015) al respecto se preguntan “¿será por lo menos cierto que esas actividades constituyen fuente indispensable para que los Estados puedan financiar políticas sociales? ¿Lo están efectivamente haciendo? ¿Y cuál es el costo para el sector público de la implantación y funcionamiento de las economías extractivas?" (pág. 8)

Por otro lado, las doctrinas neoliberales que han integrado el concepto de desarrollo local en las dinámicas por convertir el mercado en el motor de desarrollo, venciendo al vetusto Estado Benefactor, al respecto Cárdenas (2002) reseña;

Para ello es menester desubsidiar los territorios Estadales y municipales, sincerar sus potencialidades capitalistas a la luz de requerimientos de los nuevos patrones de acumulación y retornar a la valorización de "la iniciativa privada local" contrapuesta a la inercia y a la ineficiencia de las burocracias estatales y de las planificaciones centralizadas (...) Bajo el concepto central de la competitividad se plantea empujar a las ciudades locales al mercado global para competir por inversiones, capitales y tecnología que les permitan crear fuentes de trabajo para sus habitantes y preocuparse por la capacitación de sus recursos humanos, así como, por realzar ciertas cualidades que las hagan atractivas a los ojos de potenciales inversores. (pág 59-60)

En similar sentido, para Restrepo y Peña (2018) la postura neoliberal del desarrollo local se enmarca en el estímulo a la inversión extranjera directa, de la cual se esperan para el país múltiples beneficios al generar impuestos e inversión pública, principalmente en infraestructura y seguridad. Este enfoque viene acompañado de la búsqueda del desarrollo de cadenas productivas nacionales en torno a actividades primarias, al tiempo que utiliza excedentes para financiar políticas sociales y territoriales, así como la inversión en investigación en ciencia y tecnología. Para estos investigadores, los antecedentes consultados han coincidido en señalar que la reorientación hacia lo local en América Latina es una tendencia que se encuentra con grandes dificultades de diverso orden. Así, para Lucas Pozzo (2002) “En primer término, existen severas restricciones de las instituciones locales, además de la falta de una cultura local suficientemente afincada en nuestras sociedades. En segundo lugar, encontramos la crisis conceptual y metodológica de la planificación local"p12.

En concreto, sobre la precariedad de los gobiernos locales, Carballo Pozzo Ardizzi (2003, pág. 15-16) señala, por ejemplo:

a) Estructuras de financiamiento muy insuficientes.

b) Funciones múltiples y agregativas, poco compatibles con esquemas de desarrollo local.

plantaciones de monocultivo, que suelen ser manejadas por grandes empresas (Ornelas, 2016). En esta última categoría, suelen incluirse productos como la soya (o soja), el aceite de palma, los cereales y el banano, dado que se producen a gran escala, con un alto nivel de tecnología y para fines de exportación. 
c) Insuficiencias severas de personal profesional calificado y de mecanismos eficaces de selección y promoción.

d) Problemas serios de comunicación entre el municipio, las organizaciones sociales y la comunidad.

Se adiciona que, la gestión del desarrollo local se constituye en un elemento central, abordándolo desde la necesidad de generar autonomías locales a través de procesos de fortalecimiento de las sociedades regionales. Particularmente se ha estudiado el rol de los agentes y actores locales en la toma de decisiones, donde son participes el gobierno municipal y los ciudadanos, las ONGs y los empresarios, quienes puede generar incentivos para la cooperación, la responsabilidad y el mejoramiento de la gestión Carballo Pozzo Ardizzi, 2003):

El proceso local transformador requiere de la combinación de: liderazgo decisional por parte de los agentes locales más relevantes, la Alta Dirección de la ciudad; consenso organizativo, expresado en la voluntad explícita de cooperación entre gobierno, empresariado y sociedad civil local; y conocimiento pertinente, esto es, información y habilidades adecuadas a la realidad particular local generadas específicamente a partir del proceso de planificación estratégica. Este es el trípode fundamental y sinergético sobre el que se asientan los procesos locales de desarrollo. (pág. 96).

Estos aspectos, que parecen tan difíciles de lograr en la actualidad, dada la situación crítica tanto de las estructuras administrativas como de las de representación, son sin embargo una condición indispensable en cualquier estrategia de desarrollo local. A partir de estos antecedentes, el presente trabajo expondrá y analizará los efectos sobre la gestión del desarrollo local derivados de la implementación de un esquema agroindustrial extractivista, en un contexto geográfico caracterizado por la histórica dependencia económica y social con los cultivos de uso ilícito.

\section{Metodología}

Se uso un método cualitativo explicativo, con el fin de encontrar las relaciones para el fenómeno del extractivismo entre dos enfoques, ilícitio - lícito; para determinar los efectos presentados y constrastarlos. Esto para determinar los impactos en una zona vulnerable en cualquiera de los escenarios presentados. Se realizó a partir del conocimiento de la zona, a través de la observación, y la investigación exahustiva bajo una técnica deductiva que permite observar el fenómeno e ir a sus particularidades para determinar efectos y causalidades puntuales, definiendo historicamente las intervenciones de los dos modelos ejercidos en la zona.

Se estructuró una matriz para el análisis de la información recolectada; estableciendo paralelos entre lo observado empiricamente y el acervo investigativo; se realizó un filtro de selección para la relación y no relación de la misma para generar una síntesis y obtener conclusiones del estudio.

\section{Resultados}

\subsection{Contexto geográfico, económico y social del desarrollo local en Mapiripán}

\section{El aislamiento territorial y la bonanza cocalera}

La zona de estudio es uno de los territorios menos desarrollados de la Orinoquia colombiana Lemus (2005); allí no existen grandes ciudades y los municipios tienen una frágil estructuración urbana debido a las singularidades económicas y sociales de su poblamiento en distintos períodos. En particular, el sur del Meta (donde se ubica Mapiripán) ha sido una región definida como periférica, con muy poca presencia estatal, bajo acceso a la educación y a la salud y de escasa integración económica y social al resto del país. (García, Mazo \& Ramírez, 2003). 
Sin embargo, desde finales de los años 70 del siglo XX esta región fue receptora de flujos migratorios, expulsados del interior del país o atraídos por los periodos de bonaza de algunos productos de exportación, especialmente marihuana y cocaína, introducidas por grupos de narcotraficantes. De acuerdo con Lemus (2005), "la producción de cocaína se vio estimulada por el aislamiento y la relativa marginalidad espacial del área, la existencia de vías alternas de exportación (grandes ríos, pistas clandestinas de aterrizaje) y la cercanía de grandes espacios fronterizos sin vigilancia" (pág.19). Especialmente en la Orinoquia y la Amazonia se establecieron "empresas agroindustriales" de producción y procesamiento de pasta de coca, que gracias al aislamiento geográfico les fue posible el montaje de grandes laboratorios.

En particular, la llegada de la cocaína al municipio generó un proceso relativamente estable de poblamiento y ocupación del territorio, donde centenares de campesinos colonos se fundan y se vinculan al cultivo con la esperanza de mejorar sus condiciones de vida (Montenegro, 2013). Sobre esta coyuntura Arcila \& Salazar (2011) concluye que "al tratarse de un producto cuya materia prima es el follaje de una planta, era obvio que su cultivo atrajera grandes cantidades de mano de obra hacia las áreas rurales, dispuesta a participar directa e indirectamente de la bonanza" (pág .49).

En los años ochenta, las Fuerzas Armadas Revolucionarias de Colombia (FARC) entran en el municipio y se encargan de cuidar los cultivos de coca de los narcotraficantes a cambio del cobro del "gramaje", introduciendo además una estructura normativa-coercitiva que reguló las relaciones sociales y económicas entre la población civil, con lo cual la guerrilla consolidó su influencia en las economías campesinas de producción de coca. A partir de esta época, tal como lo describe Lemus (2005):

(...) a nivel social, la economía ilícita se convierte una fuente que contribuye a solventar algunas deficiencias importantes en el proceso de colonización y poblamiento de tierras baldías. Paradójicamente, frente a la política de ocupación de baldíos, sostenida por el Estado durante largo tiempo, y sus claras limitaciones institucionales y ambientales o ante los conflictos sociales que suscitó, los recursos provenientes de la economía ilícita sirvieron para sostener procesos campesinos relativamente estables de ocupación de territorios en la Orinoquia y Amazonia. (p.10)

En palabras de Arcila \& Salazar, la coca representó para la Amazonia lo mismo que el café para la región Andina de comienzos del siglo XX: su integración a una economía global mediante el cubrimiento de la demanda de un commoditie (2011). Ciertamente, con la introducción de la coca, se desencadenó un proceso de 'municipalizacion' y 'urbanizacion' en los territorios involucrados:

La casi totalidad de los actuales municipios, que anteriormente eran inspecciones de policía o corregimientos municipales, adquirieron su mayoría de edad en plena bonanza cocalera. Entre estos se cuentan municipios del sur del Meta como Puerto Rico, Uribe, Mapiripán y Puerto Concordia. (pág. 48).

Entre las particularidades de estos nuevos municipios, como lo fue el caso de Mapiripán, creado en 1989, se destaca la marginalidad gubernamental y desconfianza en las instituciones del Estado local y departamental, "contexto agudizado por la situación predominante de conflicto armado ha determinado la fragilidad de la soberanía estatal en buena parte del Meta”. (Lemus, 2005, pág. 16).

Así mismo, vale la pena mencionar que con la colonización cocalera se ahondaron graves procesos de deterioro ambiental. En efecto, los cultivos ilícitos son uno de los principales problemas ambientales que enfrenta el país:

(...) ya que presionan los bosques, destruyen los ecosistemas, la biodiversidad y el capital social y humano. Se estima que por cada hectárea de coca sembrada se destruyen dos hectáreas de bosque, y por cada hectárea de 
amapola se destruyen a su vez 2,5, afectando especialmente el ecosistema alto andino y la región amazónica. (García, Mazo \& Ramírez, 2003, pág.4)

Según la investigación de Montenegro (2013), a mediados de los años noventa, Mapiripán llega a ser uno de los municipios con mayor nivel de producción y área sembrada de coca, como resultado del establecimiento de grandes laboratorios de producción. Paralelamente, el Gobierno nacional lanzó una política de erradicación de cultivos ilícitos que combinaba diversas formas de erradicación forzada y voluntaria. En este marco se creó en 1994 el programa Plan Nacional de Desarrollo Alternativo (PLANTE) con el fin de complementar las campañas de erradicación forzosa, mediante inversiones de carácter social para prevenir, frenar y eliminar la producción de cultivos ilícitos, limitándose a zonas de economía campesina e indígena (García, Mazo \& Ramírez, 2003).

A finales de los años noventa Mapiripán "era un corredor estratégico del narcotráfico por su privilegiada ubicación geográfica sobre el rio Guaviare, con salida al Orinoco". (Montenegro, 2013, pág. 38).

Así, para el año 2000:

La principal fuente de empleo en el municipio es la producción, explotación y comercialización de coca que genera alrededor de 150.000 jornales anuales en 5.000 has de coca (...) Por razones de orden público ocasionado por grupos armados al margen de la ley, la comercialización del ganado y de los productos agrícolas es bastante limitada, ya que esta se ha venido restringiendo paulatinamente. (EOT, 2000, pág. 122)

A la par, García, Mazo \& Ramírez (2003) encontró que en las zonas cocaleras;

Los altos ingresos obtenidos por el cultivo, generaron un proceso de transformación social, reflejado en un consumo desmedido, prostitución falta de previsión para el futuro y pérdida de identidad como comunidad rural, condiciones que sumadas a la presencia de grupos subversivos, han empeorado la situación.(pág. 2)

Mostrando otra faceta, el ingreso de nuevos actores armados ilegales que buscaron disputar el territorio con las FARC. Entre ellos las Autodefenzas Unidas Campecinas (AUC), grupo paramilitar que a partir del año 2000 comenzó a ganar cada vez más control de las áreas dedicadas al cultivo de coca. De hecho, en 2001 el narcotraficante Miguel Arroyave le compró por siete millones de dólares el Bloque Centauros de las AUC a la casa Castaño. A partir de entonces el grupo se orientó hacia el control de las crecientes áreas de coca en el departamento (González Posso, 2007; Montenegro, 2013). El control territorial de los grupos paramilitares se prolongaría de forma notoria hasta el año 2011, fecha en la cual se consolidó la crisis de la económica cocalera, lo cual golpeo notoriamente al poblador rural y urbano de Mapiripán, para quienes solamente había existido la alternativa comercial que ofrece el cultivo y el procesamiento químico de la coca. (Lemus, 2005).

\section{Escenario político, económico y social de Mapiripán en 2008}

Al momento de los primeros contactos de los inversionistas de Poligrow con Mapiripán, en 2008, el municipio se caracterizaba por grandes problemas de gobernabilidad y debilidad organizativa de la sociedad civil. (Lemus, 2005). Además, la economía rural se encontraba en crisis reciente por las decisiones de los grupos armados ilegales y por los efectos no previstos de la aplicación de la política de lucha contra las drogas y el Plan Colombia. Desde el aspecto social, las familias de esta zona cocalera, cuyos integrantes son predominantemente jóvenes y casi la mitad mujeres, enfrentaban múltiples condiciones de vulnerabilidad; sobre este asunto la Fundación Ideas para la Paz y la Oficina de las Naciones Unidas contra la Droga y el Delito (UNODC) encontraron que:

Contrario a la idea de que participar en esta economía ilícita es rentable, lo que muestran los resultados de la encuesta es que los niveles de desarrollo y calidad de vida de las familias involucradas en el cultivo 
están muy por debajo si se les compara con la población rural en Colombia. Estas familias enfrentan importantes brechas sociales y económicas, en medio de un ciclo de exclusión y pobreza. (...) En cuanto a la pobreza monetaria extrema, el $35 \%$ de los hogares que están en zonas de cultivos de coca se encuentran en esta categoría, más del doble de lo registrado en los centros poblados y zonas rurales dispersas, donde según información del DANE este porcentaje es del 15,4\%. (UNODC-FIP, 2018, pág. 911)

Para aquella época se evidencia que los desafíos para el Estado eran múltiples y demandaban voluntad política, recursos y tiempo. Se consideraba que la superación de estos problemas no competía solo al Gobierno Nacional, sino que debía involucrar a las comunidades afectadas, las autoridades locales y también al sector privado.

\section{Consolidación de los incentivos del Gobierno Nacional al sector agroindustrial de la palma de aceite}

En medio de esta coyuntura, el gobierno nacional resolvió apoyar enérgicamente al cultivo de Palma de aceite, sector con gran trayectoria en el país. Introducida por primera vez en 1932, pero solamente hasta 1945 empieza su cultivo comercial por parte de la United Fruit Company en el departamento de Magdalena (Indepaz, 2013).

A partir de entonces el crecimiento del cultivo es regular, pero relativamente lento en comparación con países como Indonesia o Malasia. Desde sus orígenes, el cultivo de palma cuenta con un apoyo sistemático del gobierno y la gestión gremial de la Federación Nacional de Cultivadores de Palma de Aceite (Fedepalma; Bozzi, 2013; Montenegro, 2013).

Este momento de consolidación, rastrea sus antecedentes directos en 2001, cuando el presidente Pastrana viaja a Malasia e invita a los empresarios palmeros de ese país a invertir en palma de aceite en Colombia:

Así mismo, desde el principio de su gobierno en 2002, el presidente Uribe asume la producción de biocombustibles como uno de los ejes estratégicos de su política agropecuaria (Ahumada Rueda, 2013; Mingorace et all, 2004). Álvaro Uribe llega la presidencia con la idea y decisión de promover los biocombustibles (Mesa, 2009). Durante los dos gobiernos de Álvaro Uribe (2002-2010) se otorgan variados estímulos tributarios y fiscales al sector palmero. También se otorga acceso a tierras y otros factores productivos. (Indepaz, 2013). // El presidente Santos parece haber ratificado su compromiso con el avance de la actividad palmera al haber afirmado: "al terminar nuestro gobierno, en el 2014, esperamos haber superado la meta de 600 mil hectáreas cultivadas, con lo que Colombia seguirá consolidándose como el primer productor de aceite de palma en el continente americano" (Montenegro, 2013, pág. 27-28).

En 2007 el gobierno define su estrategia para el desarrollo del sector palmero a través del documento Conpes 3477 de julio de 2007. De acuerdo con Montenegro (2013), el objetivo de política definido en el documento es: "incrementar la competitividad y la producción de la agroindustria palmera, en forma económica, ambiental y socialmente sostenible, aprovechando las ventajas del país y el potencial de un mercado creciente, con el fin de ofrecer nuevas oportunidades de desarrollo, empleo y bienestar en las zonas rurales" (Conpes, 2007). Posteriormente se define la estrategia del gobierno para promover la producción de biocombustibles a través del documento Conpes 3510 de 2008.

En 2008 por gestión de ProColombia se facilitó y acompañó la llegada de la compañía italo-española Poligrow, firma que buscaba una zona en la altillanura para sembrar palma de aceite con un proyecto a más de 60 años y con una inversión a precio 2019 de más de US\$70 millones de dólares. (Diario de Occidente, 2019, 21 de abril). Desde su ingreso, la empresa ha sido duramente cuestionada porque las tierras en donde opera tienen un pasado turbulento (El Espectador, 2018); 
En primer lugar, el dueño inicial de la hacienda Macondo fue un campesino antioqueño que abandonó su tierra y salió de la región en 1999, en plena época de la violencia paramilitar. Desde entonces, aunque el campesino ha asegurado que no vendió sus tierras, estas pasaron por varios dueños, antes de ser compradas por Poligrow en 2009. (Montenegro, 2013, pág. 42).

Desde aquellos años en Colombia se ha generado un intenso debate en torno al desarrollo de los cultivos de palma de aceite. En términos generales se ha discutido sobre los impactos medioambientales de la actividad y la idea de que existen relaciones entre el cultivo y los actores armados, en particular, los paramilitares. Al respecto, existen trabajos de investigación que exploran la relación entre el cultivo de palma de aceite y el conflicto armado en el municipio de Mapiripán, de acuerdo con la hipótesis de que la violencia paramilitar en el municipio ha sido un factor que ha posibilitado la implantación del cultivo (Montenegro, 2013), a modo de ejemplo, los investigares de este tema han abordado también el crecimiento del sector palmero en otras regiones del Colombia;

En la región del Bajo Atrato chocoano, se ha comprobado que empresarios palmeros, políticos y militares colaboraron con grupos paramilitares en el despojo a comunidades negras de sus tierras para sembrar palma (Franco y Restrepo, 2011). Por estos hechos la justicia colombiana ha condenado varios políticos y empresarios (Corte Suprema de Justicia, 2013). Igualmente, en regiones como Tumaco el cultivo ha estado vinculado a violaciones de los derechos humanos, desplazamiento forzado y ocupación ilegal de tierras (Indepaz, 2013, Segura 2008). Otros estudios han sostenido que el cultivo ha incidido en procesos de desplazamiento (Goebertus, 2008). En contraste, hay estudios que desmienten el vínculo entre la palma y el conflicto y sugieren que la mayoría de acciones violentas de los grupos armados han ocurrido en regiones distintas a las palmeras (Rangel, Tobón y Betancur, 2009). (Montenegro, 2013, pág. 1)

Pese a las múltiples acusaciones, la multinacional Poligrow continuó con su operación. De esta forma, en 2010, la firma palmicultora creó la Fundación Poligrow a través de la cual desarrolló programas y proyectos en la comunidad, enfocados en diversas líneas de acción como educación, fortalecimiento de proyectos productivos, calidad de vida, convivencia, valores, cultura de la legalidad, entre otros (Diario de Occidente, 2019). Así mismo, la continuidad de la expansión y consolidación de Poligrow ha sido asegurada a largo plazo por ejemplo, en 2018 se anunció:

La inversión de 18 millones de dólares para ampliar y modernizar su planta de extracción de aceite de palma en el municipio de Mapiripán, Meta. Por otra parte, el crecimiento de Poligrow lo marcará también el incremento de 7.000 a 15.000 hectáreas sembradas con palma aceitera. Las áreas se consolidarán a través de negociaciones con inversionistas y productores particulares, a través de esquemas como agricultura por contrato o a través de participaciones y alianzas empresariales, informó la empresa. (EI tiempo, 2018)

Para 2019, Colombia se consolidó como el cuarto productor de aceite de palma del mundo y el primero en América, con cerca de 500.000 hectáreas sembradas que generan más de 140.000 empleos directos e indirectos. En el caso de Mapiripán, el aceite de palma se convirtió en el pilar de la economía y es el responsable de la transformación social y económica del municipio. (Diario de Occidente, 2019). En otras palabras, la presencia agroindustrial del cultivo permitió que las condiciones socioeconómicas de la población de Mapiripán hayan cambiado radicalmente, ya que el mismo le aporta $80 \%$ a la economía del municipio, mostrando alternativas laborales y de mejora en su calidad de vida (Agronegocios, 2018). Al respecto, los diversos testimonios de pobladoresde Mapiripán resaltan los cambios ocurridos:

Se comenzó a vivir una nueva era en la legalidad, atrás quedaron los cultivos ilícitos y hoy, más del $80 \%$ de la fuerza laboral de Mapiripán está empleada por Poligrow en labores de siembra, recolección y 
producción de aceite de palma, con más de 650 plazas laborales. Así lo manifiesta Luis Ángel Rivera, operario en cosecha y recolección de fruto de palma, 'antes, lo que para nadie es un secreto, se trabajaba con cultivos ilícitos y ese era el ingreso del municipio, viene la erradicación por parte del Estado y posteriormente llega Poligrow, cuyo proyecto trajo un cambio extremo, un árbol frondoso que brinda sombra, oportunidades, empleo.

La docente María Isabel Mendivelso, quien afirma que: 'Un indicador de progreso es que hoy más niños y jóvenes asisten a los colegios de forma permanente, también lo hacen los de las comunidades indígenas Jiw y Sikuani, que antes permanecían en las calles. Esto como resultado de nuevas condiciones de empleo y estabilidad de sus padres, sumado a la desaparición del conflicto y una nueva cultura de legalidad. Se recuperó la familia y con ella la educación de Mapiripán (Diario de Occidente, 2019).

Por otro lado, ante la necesidad de los inversionistas de generar un negocio que ofrezca rentabilidad económica, se han iniciado procesos de acercamiento y conciliación con la comunidad para identificar las expectativas y necesidades de la población, buscando de manera estratégica evitar demandas judiciales y administrativas, causadas por conflictos sociales o medio ambientales. A pesar de que se presentó falencia en los procesos de consulta previa para diseñar socializar con la participación de todos los actores, los procesos de comunicación se han fortalecido paulatinamente entre los diferentes actores (Rodríguez González, 2014).

Para Jersson Espinosa, coordinador de la Casa de la Cultura del municipio, Mapiripán vive un cambio reflejado en las condiciones de vida de su gente. 'Hoy las casas son de material, ladrillo, cemento, pintura, lo que sería normal en otra ciudad, en el pasado eran de bareque y eso es reflejo del ingreso y generación de empleo en la población. Mapiripán ha surgido de la oscuridad a la luz'. Literalmente, como lo manifiesta Espinosa, el que es el segundo municipio más grande del departamento del Meta y uno de los 20 con mayor potencial agroindustrial por su extensión en Colombia, pasó de las tinieblas a la luz. (Diario de Occidente, 2019).

Antes de 2011 los mapiripenses contaban con menos de 8 horas de energía eléctrica al día. Como resultado de una alianza realizada por Poligrow, el Ministerio de Minas y Energía con el apoyo de la Gobernación del Meta se crea la electrificadora de Mapiripán - Electrimapiri, permitiendo el suministro a la población de fluido eléctrico las 24 horas al día. 'La apuesta que hizo esta multinacional por Mapiripán ha contribuido a mejorar los entornos, a la reactivación del comercio, y al comienzo de la llegada de turistas. El contar con energía 24 horas ha transformado la dinámica diaria de la vida de todos', afirma Ricardo Jara, director técnico de Electrimapiri. (Diario de Occidente, 2019, 21 de abril).

Con la palma llegó la paz, el empleo y la energía eléctrica, en un momento en el que nueva inversión está llegando, se están empezando a pavimentar calles, se abren nuevos locales comerciales y más construcciones de casas y los hoteles han ampliado el número de habitaciones, como el caso de La Posada de Lucy que pasó de cuatro a 16 en los últimos años sostiene su propietaria, Lucy Miranda de Carrero. (Diario de Occidente, 2019, 21 de abril).

A nivel local, Poligrow ha sabido constituir una alianza con la Alcaldía de Mapiripán, recibiendo créditos de 55,3 millones de pesos colombianos (COP) para desarrollar obras de mejora de vías. Al mismo tiempo, por su lado, la Fundación Poligrow conjuntamente con Electrimapiri, promueve el proyecto "Autoabastecimiento de energía a partir de aceite vegetal en el Municipio de Mapiripán", financiado por el Banco Interamericano de Desarrollo y algunas agencias de cooperación alemana y coreana (Finzi, 2017, pág. 40), inclusive, durante un acto de conmemoración de los 20 años de la masacre de Mapiripán, Meta, Mónica Espinosa Valencia, delegada del comité departamental de víctimas de ese municipio. 
Una de las entidades que ha visitado esta zona con frecuencia es el Centro de Estudios de la Orinoquía (CEO) de la Universidad de los Andes, apreciando el impacto que ha tenido en la población de Mapiripán el desarrollo de iniciativas agroindustriales en terrenos calificados con anterioridad como no fértiles para el sector agro. El director del CEO, Carlos Montenegro, expresó que "teníamos un gran interés en analizar cómo una compañía extranjera podía generar una transformación allí y apreciar como lo recibían sus habitantes". Fue así que la entidad descubrió en diversas visitas como con el impacto del cultivo de palma de aceite "se compenetró una transformación sobre cómo los pobladores debían hacerlo y asumir esta actividad productiva. Se preocuparon por fortalecer su comunidad y proteger el medio ambiente" (Agronegocios, 2018).

Sin embargo, pese a los beneficios descritos, se ha presentado una transformación en la tenencia de la tierra como consecuencia de que la implementación de este modelo de negocio exige la utilización y en su defecto la compra de grandes extensiones de suelo, incrementando la problemática de la concentración de tierra y de medios de producción. Por último, se presenta un condicionamiento de la soberanía como resultado de la implementación de un modelo de desarrollo que a pesar de generar más beneficios que problemáticas, es impuesto sin consultar con la población su implementación. (Rodríguez, 2018)

Ciertamente, la empresa ha sabido construir una densa red de alianzas estratégicas multidimensionales que le han permitido legitimar el proyecto tanto a niveles locales, nacionales e internacionales, muestra de ello, desde la Expo Milano 2015 fortaleció sus vínculos institucionales tanto en Italia como en Colombia. En efecto, durante la visita oficial a Colombia del Primer Ministro italiano Matteo Renzi, el mismo Carlo Vigna Taglianti reconoce haber participado en un encuentro privado con el Presidente Juan Manuel Santos (Comisión Intereclesial Justicia y Paz, 2016), lo que testimonia el altísimo nivel de inserción en los circuitos institucionales del Director General de la empresa Poligrow. (Finzi, 2017, pág. 40-41).

De este modo, para 2019, el extractivismo agroindustrial como modo de gestión del desarrollo local en el municipio de Mapiripán (Meta), mostró generar efectos positivos producto de la dinamización de la economía. Por un lado, es generada principalmente por la contratación de personal de forma directa con el sector privado, teniendo como efecto principal la liquidez de dinero efectivo de los pobladores del municipio, que involucra adicionalmente, la generación de otras fuentes de ingresos anexos al cultivo de palma, que ha permitido mejorar indicadores de desempleo y subempleo. Lo anterior se manifiesta en la formalización de empleo con prestaciones laborales. (Calderón Uribe, 2018)

Extractivismo privado industrial, como alternativa de contención frente a la amenaza actual del regreso a los cultivos de uso ilícito:

Con la creación del Programa Nacional Integral para la Sustitución de cultivos de uso ilícito (PNIS), como resultado del acuerdo de paz entre gobierno y las Farc, los campesinos vieron la oportunidad de reemplazar la coca por alguna otra labor legal y que no les represente problemas con grupos armados que controlan este negocio. (Semana rural, 2019), en opinión de (González, 2017) :

El Plan de Acción Inmediata se plasmó en compromisos escritos que tienen varios componentes de corto plazo y con un horizonte final de dos años. El primer componente de apoyo a las familias con pagos mensuales o bimensuales para un total de $\$ 12$ millones en el primer año, más $\$ 1,8$ millones para proyecto de pan coger y dos proyectos productivos, de ciclo corto y largo plazo, por valor de \$19 millones y además $\$ 3,8$ millones/familia para asistencia técnica. A ese paquete de $\$ 36$ millones/familia se le agregan otros aportes de bienestar social urgente al adulto mayor, infantes, atención en salud y educación, apoyo para búsqueda de empleo de los asalariados y de créditos o recursos para proyectos asociativos, planes de infraestructura de ejecución rápida, de sostenibilidad y protección ambiental. El total puede estimarse en $\$ 60$ millones de pesos familia en el primer año, sin 
incluir la formalización o acceso a la tierra en forma progresiva y el inicio del conjunto de planes territoriales previstos en el Acuerdo Final y en las políticas públicas de inversión social. Esto significa que sólo el PAl, entendido como la cuota inicial de los Programas Integrales de Sustitución tendría un valor de \$24 billones de pesos si se pretendiera atender a las 100.000 familias que están cubiertas por los pactos ya firmados y a otras 100.000 que están pidiendo ser incluidas. ( p.4)

Según cifras del Programa Nacional Integral para la Sustitución de Cultivos llícitos (PNIS, 2018), en Mapiripán se encuentran vinculadas 700 familias, que reportaron 846 hectáreas de coca. Se reporta el asesinato de la lideresa María Magdalena Cruz Rojas y el desplazamiento de 6 familias vinculadas al PNIS, por presiones de las disidencias de las Farc. Claramente, el incumplimiento de lo acordado no solo tiene implicaciones en la confianza y credibilidad de las instituciones, sino que genera un alto riesgo de resiembra y el posible aumento de las hectáreas de coca (FIP, 2018, pág. 34). Muestra de los altos riesgos de recultivo es el incremento de las hectáreas de coca; mientras en 2016 el número de hectáreas cultivadas llegaba a 146.000, en 2017 alcanzó la cifra histórica de 209.000, lo que representa un incremento de cerca del 43 \%, según cálculos de la Oficina de Política Nacional para el Control de Drogas de la Casa Blanca (El Espectador, 2018). Así las cosas, los proyectos productivos de sustitución se están quedando en el papel. Los retrasos en los pagos de los subsidios para quienes dejaron de cultivar, las amenazas de seguridad contra los verificadores de la sustitución y las dificultades técnicas para comenzar proyectos productivos son obstáculos de este proceso desafortunadamente es cada vez mayor la distancia entre la demanda por la implementación de los pactos voluntarios y la capacidad o velocidad de respuesta que ha mostrado el gobierno. (González, 2017, pág. 4).

\section{Conclusiones}

La colonización cocalera, entendida como un fenómeno de naturaleza móvil y temporal, propia de las bonanzas extractivistas, impulsó un proceso de desarrollo local en zonas aisladas geográficamente, donde los organismos gubernamentales tuvieron una mínima participación. De este modo, el extractivismo pasajero de la bonanza cocalera dejó tras de sí un poblamiento permanente que queda a la deriva ante la falta de opciones de sustitución que se acerquen a las rentabilidades de la economía cocalera.

Ante las dificultades y retos para la viabilidad de los programas de sustitución de cultivos de uso ilícito, la inversión agroindustrial en zonas aisladas y sin infraestructura, ha puesto a prueba en el caso de Mapiripán un modelo de desarrollo local basado en la inversión privada, que ha elaborado proyectos de gestión social y de infraestructura. Así las cosas, en estas zonas de frontera agrícola se pasa de un extractivismo a otro, donde la legalidad da respaldo y sostenibilidad en el tiempo a los nuevos productos agroindustriales, aunque estos menoscaben la diversificación productiva y la autonomía política del municipio.

En este caso, las relaciones y cooperación para la gestión del desarrollo local entre empresa privada, organismos gubernamentales y comunidades, presenta una fuerte dependencia del actor - agente empresarial, quien, por tanto, define el camino a seguir. Así, la lectura de la realidad local en Mapiripán refleja la materialización de la estrategia Neoliberal el Desarrollo Local, como lo señala Cárdenas (2002), “ (...) crear fuentes de trabajo para sus habitantes y preocuparse por la capacitación de sus recursos humanos, así como, por realzar ciertas cualidades que las hagan atractivas a los ojos de potenciales inversores". ( pág .59).

Respecto a este punto, Pozzo (2002, pág. 6) señala que "uno de los grandes ejes de problemas a resolver en este contexto para la gestión del desarrollo local son los vinculados con la necesidad de aumentar la capacidad técnica y de gestión de los gobiernos municipales". Por tanto, es fundamental un cambio profundo, que tiene que ver con la lógica de la acción de sus agentes, y en el cual la transparencia, la eficiencia y la oportunidad deben ser pilares fundamentales de una nueva forma de gestionar a nivel local. 


\section{Referencias bibliográficas}

Agronegocios. (2018). Poligrow Colombia proyecta completar la siembra de 15.000 hectáreas de palma. https://www.agronegocios.co/agricultura/cuantas-hectareas-de-palma-de-aceite-hay-en-mapiripan2756390

Alimonda, H. (2015). Provocaciones sobre el tema "Extractivismo y Desarrollo". Polis. Revista Latinoamericana, (41).

Arcila, O. H., \& Salazar, C. A. (2011). La Amazonia colombiana: poblada y urbanizada. Revista Colombiana Amazónica, 4, 37-55.

Bozzi, M. L. O. (2013). Palma de aceite en Colombia. Villegas Editores.

Calderón Uribe L. (2018). La responsabilidad social empresarial de la actividad agroindustrial en Colombia: el caso de la multinacional Poligrow.

Carballo Pozzo Ardizzi, L. (2003). Los nuevos desafíos para la gestión del desarrollo local. Millcayac. Anuario de Ciencias Políticas y Sociales, 1(1).

Cárdenas, N. (2002). El desarrollo local su conceptualización y procesos. Provincia, núm. 8, enero-junio, 2002, pp. 53-76. Universidad de los Andes. Mérida, Venezuela. Disponible en: http://www.redalyc.org/articulo.oa?id=55500804

Cardoso, F. H., \& Faletto, E. (1996). Dependencia y desarrollo en América Latina: ensayo de interpretación sociológica. Siglo xxi.

CONPES- Consejo Nacional de Política Económica y Social (2007). Documento Conpes 3477, estrategia para el desarrollo competitivo del sector palmero colombiano. Departamento Nacional de Planeación. Consejo Nacional de Política Económica y Social.

CONPES. (2008). Documento Conpes 3510, lineamientos de política para promover la producción sostenible de biocombustibles en Colombia. Departamento Nacional de Planeación. Consejo Nacional de Política Económica y Social.

Diario de Occidente. (2019). Mapiripán, el renacer de la esperanza. https://occidente.co/nacionales/mapiripanel-renacer-de-la-esperanza/

El Tiempo Sección Economía y Negocios 5 de enero de 2018. Agricultura por contraro

Economía hoy. (2017). Víctimas de Mapiripán destacan aporte socioeconómico de ítalo española Poligrow. consultado el 08 mayo 2019. https://www.economiahoy.mx/noticias/noticias/8511439/07/17/Victimasde-Mapiripan-destacan-aporte-socioeconomico-de-italo-espanola-Poligrow.html

El Espectador. (2018). Sustitución voluntaria de cultivos de uso ilícito, en veremos. https://www.elespectador.com/colombia2020/pais/sustitucion-voluntaria-de-cultivos-de-uso-ilicito-enveremos-articulo-857033

El Tiempo. (2018). Poligrow invertirá US\$ 18 millones en planta extractora. consultado el 08 mayo 2019. https://www.eltiempo.com/economia/empresas/poligrow-invertira-us-18-millones-en-planta-extractoraen-el-meta-168420

EOT- Esquema de Ordenamiento Territorial. (2000). alcalde Conrado Salazar Cardona. 
Frank, A. G., Pacios, E., \& Izaguirre, I. (1970). Capitalismo y subdesarrollo en América Latina (No. 330.98/F82cE). Editorial de ciencias sociales.

Finzi, G. (2017). El caso de Poligrow en Mapiripán, Meta: entre acaparamiento (ilegal) de tierras y capitalismo verde. Ciencia Política, 12(24), 21-50.

FIP- Fundación Ideas para la Paz. (2018) ¿En qué va la sustitución de cultivos ilícitos? La implementación, los rezagos y las tareas pendientes. Fundación Ideas para la Paz -FIP-. Bogotá, enero - marzo 2018. http://ideaspaz.org/media/website/FIP_sustitucion_final.pdf

Furtado, C. (1971). Dependencia externa y teoría económica. El trimestre economico, 38(150 (2), 335-349.

García, L. A., Mazo, I., \& Ramírez, A. M. (2003). Propuesta para la sustitución de cultivos ilícitos mediante

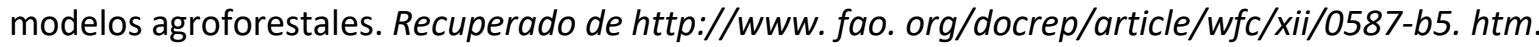

González Posso, C. (2017). Balance de un año de implementación de la política de sustitución de cultivos de coca. http://www.indepaz.org.co/balancede-un-ano-de-implementacion-de-la-politica-de-sustitucion-decultivos-de-coca/

Lemus, O. G.(2005) análisis de la economía política de la coca en el departamento del meta 1982-2004.Estudio financiado por la Agencia Sueca de Cooperación Internacional para el Desarrollo, ASDI.

Alimonda, H. (2015). Provocaciones sobre el tema "Extractivismo y Desarrollo". Polis [En línea], 41 | 2015, Publicado el 19 septiembre 2015, consultado el 09 mayo 2019. URL : http://journals.openedition.org/polis/10925

Indepaz - Instituto de estudios para el desarrollo y la paz. (2013). Informe especial observado. http://www.indepaz.org.co/observatorio-cultivos-y-cultivadores-declarados-ilicitos/

Marini, R. M. (1979). Plusvalía extraordinaria y acumulación de capital. Cuadernos políticos, 20, 18-39.

Montenegro Perini, G. (2013). Agroindustria y conflicto armado en el meta: palma de aceite en el municipio de Mapiripán (1997-2013). Trabajo de grado. Pontificia Universidad Javeriana, facultad de ciencias políticas y relaciones internacionales, carrera de ciencia política. Bogotá D.C.

PNIS- Programa Nacional Integral para la Sustitución de Cultivos (2018). Ilícitos, estado de avance. http://www.camara.gov.co/sites/default/files/201810/Anexo\%202.\%20DIRECCI\%C3\%93N\%20PARA\%20LA\%20SUSTITUCI\%C3\%93N\%20DE\%20CULTIVOS\%2OIL ICITOS.PROPOSICI\%C3\%93N\%20072\%20-\%202018_0.pdf

Restrepo Botero, D. I., \& Peña Galeano, C. A. (2017). Territorios en disputa: Tensiones entre "extractivismo", derechos étnicos, gobiernos locales y medio ambiente en Bolivia, Colombia, Ecuador y Perú. International Development Policy/ Revue internationale de politique de développement, 9(9).

Rodríguez González, I. (2014). Dispossession, wastelands and armed conflict in Puerto Gaitán and mapiripán (Meta, Colombia) between 1980 and 2010. Estudios Socio-Jurídicos, 16(1), 289-314.

Rodríguez, E. C.(2018). ¿ Contra las drogas o por las tierras?: la política prohibicionista de drogas como motor de despojo de tierras y destrucción de la biota en el sur de Colombia.

UNODC, iFIP- Fundación Ideas para la Paz y la Oficina de las Naciones Unidas contra la Droga y el Delito. (2018) ¿Quiénes son las familias que viven en las zonas con cultivos de coca? Caracterización de las familias beneficiarias del Programa Nacional Integral de Sustitución de Cultivos Ilícitos (PNIS). 